\title{
Reversal of established autoimmune diabetes by restoration of endogenous $\beta$ cell function
}

\author{
Shinichiro Ryu, ${ }^{1}$ Shohta Kodama, ${ }^{1}$ Kazuko Ryu, ${ }^{1}$ David A. Schoenfeld, ${ }^{2}$ \\ and Denise L. Faustman ${ }^{1}$ \\ ${ }^{1}$ Immunobiology Laboratory, and \\ ${ }^{2}$ Department of Biostatistics, Harvard Medical School and Massachusetts General Hospital, Charlestown, Massachusetts, USA \\ Address correspondence to: Denise L. Faustman, Immunobiology Laboratory, Massachusetts General Hospital-East, \\ Harvard Medical School, Building 149, Room 3602, 13th Street, MailStop M1493601, Charlestown, Massachusetts 02129, \\ USA. Phone: (617) 726-4084; Fax: (617) 726-4095; E-mail: denise.faustman@cbrc2.mgh.harvard.edu. \\ Shinichiro Ryu and Shohta Kodama contributed equally to this work. \\ Received for publication January 24, 2001, and accepted in revised form May 14, 2001.
}

In NOD (nonobese diabetic) mice, a model of autoimmune diabetes, various immunomodulatory interventions prevent progression to diabetes. However, after hyperglycemia is established, such interventions rarely alter the course of disease or allow sustained engraftment of islet transplants. A proteasome defect in lymphoid cells of NOD mice impairs the presentation of self antigens and increases the susceptibility of these cells to TNF- $\alpha$-induced apoptosis. Here, we examine the hypothesis that induction of TNF- $\alpha$ expression combined with reeducation of newly emerging T cells with self antigens can interrupt autoimmunity. Hyperglycemic NOD mice were treated with CFA to induce TNF- $\alpha$ expression and were exposed to functional complexes of MHC class I molecules and antigenic peptides either by repeated injection of MHC class I matched splenocytes or by transplantation of islets from nonautoimmune donors. Hyperglycemia was controlled in animals injected with splenocytes by administration of insulin or, more effectively, by implantation of encapsulated islets. These interventions reversed the established $\beta$ cell-directed autoimmunity and restored endogenous pancreatic islet function to such an extent that normoglycemia was maintained in up to $75 \%$ of animals after discontinuation of treatment and removal of islet transplants. A therapy aimed at the selective elimination of autoreactive cells and the reeducation of $T$ cells, when combined with control of glycemia, is thus able to effect an apparent cure of established type 1 diabetes in the NOD mouse.

J. Clin. Invest. 108:63-72 (2001). DOI:10.1172/JCI200112335.

\section{Introduction}

Autoimmune destruction of pancreatic $\beta$ cells is more than $90 \%$ complete by the time hyperglycemia becomes clinically evident in individuals with type 1 (insulindependent) diabetes mellitus. Prevention of this disease would therefore optimally require arrest of autoimmunity in the prehyperglycemic phase. After hyperglycemia is established, therapies based on islet cell replacement are necessary to restore physiological control of blood glucose. Although islet transplantation has been successful in mice, rats, and nonhuman primates with chemically induced diabetes, sustained survival of allogeneic islet grafts is infrequently observed in spontaneous diabetic hosts such as the NOD (nonobese diabetic) mouse, BB (BioBreeding) rat, and diabetic humans (1-5). Thus, allogeneic or xenogeneic cellular grafts subjected to transient ablation of donor MHC class I antigen expression (6) - achieved either with the use of "masking" Ab's to MHC class I molecules or by deletion of the $\beta_{2}$-microglobulin $\left(\beta_{2} \mathrm{M}\right)$ gene - are capable of permanent engraftment in nonautoimmune recipients, but are minimally protected from recurrent $\beta$ cell autoimmunity in NOD mice (7,
8). The immune mechanisms of islet graft rejection and recurrent autoimmunity appear distinct, and protective interventions targeted at these two pathways of islet destruction are nonoverlapping in effectiveness.

Subsets of antigen-presenting cells and $\mathrm{T}$ cells of NOD mice that progress to hyperglycemia exhibit a decrease in the production of LMP2, a catalytic subunit of the proteasome, after about 5-6 weeks of age $(9,10)$. This defect is accompanied by deficient generation of peptides from endogenous proteins for display on the cell surface by MHC class I molecules, a process that is important for $\mathrm{T}$ cell memory and tolerance to self antigens $(11,12)$ and is impaired in various human and murine autoimmune diseases $(11,13,14)$. The proteasome also contributes to the processing and activation of NF- $\mathrm{KB}(15-17)$, a transcription factor that regulates the expression of genes that contribute to cytokine generation, lymphocyte maturation, protection from TNF$\alpha$-induced apoptosis, and promotion of the processing of antigens for presentation by MHC class I molecules $(18-20)$. The proteasome defect in NOD mice affects lymphoid maturation as a result, at least in part, of impaired activation of NF- $\mathrm{KB}$. 
In normal humans and other mammals, the continuous expression of MHC class I molecules by peripheral cells, including islet cells (21), maintains peripheral tolerance in the context of properly selected lymphocytes $(12,22)$. Interruption of exposure to complexes of self peptides and MHC class I molecules results in the aberrant selection of $\mathrm{CD}^{+} \mathrm{T}$ cells that exhibit an increased sensitivity to apoptosis (23). Diabetic humans and NOD mice that progress to diabetes thus manifest a paucity of memory cells and an increased susceptibility of $\mathrm{T}$ cells to apoptosis, traits that may be secondary, in part, to improper presentation of self antigens by MHC class I molecules and ineffective $\mathrm{T}$ cell selection $(10,11)$.

On the basis of the view that islet transplantation into hyperglycemic NOD mice will require both the prevention of transplant rejection and the elimination of autoimmune $\mathrm{T}$ cells, we sought to combine two strategies to achieve these goals. To bypass graft rejection, we used donor islets from C57BL/6 mice in which the $\beta_{2} \mathrm{M}$ gene was deleted. MHC class I proteins are re-expressed on graft cells within 24 to 72 hours after transplantation as a result of reconstitution with host $\beta_{2} \mathrm{M}$ present in plasma $(6,24,25)$. The re-expression of donor MHC class I antigens is important because it is necessary for the development and maintenance of peripheral tolerance.

With regard to interruption of autoimmunity, we hypothesized that the lineage-specific defects both in peptide presentation by $\mathrm{MHC}$ class I molecules and in the processing and activation of NF- $\mathrm{KB}$ might be important in the pathogenesis of diabetes in NOD mice. The $\mathrm{NF}-\kappa \mathrm{B}$ defect in the affected lineages on NOD mice is accompanied by an increased sensitivity of these cells to TNF- $\alpha$-induced apoptosis in vitro (10). The increased susceptibility to apoptosis of misselected $\mathrm{T}$ cells that result from improper education by $\mathrm{MHC}$ class I peptide complexes suggested that the production of TNF- $\alpha$ in vivo might promote the selective death of such poorly educated lineages $(10,26,27)$. Furthermore, treatment of diabetic NOD mice or BB rats with CFA, an inducer of TNF- $\alpha$ production, both impairs the transfer of disease by $\mathrm{T}$ cells from these animals to naive hosts (28-31) as well as prolongs the survival of syngeneic islet grafts in spontaneously diabetic hosts $(2,32)$. We therefore hypothesized that CFA treatment might eliminate, at least temporarily, the autoreactive lymphoid cells of NOD mice by promoting their apoptosis, in part through the induction of TNF- $\alpha$.

Thus, we both treated hyperglycemic NOD recipients with CFA, seeking to eliminate autoreactive lymphoid lineages, and transplanted islets from $\beta_{2} \mathrm{M}$-deficient donors under the kidney capsule of these animals, seeking to generate graft-specific tolerance. These interventions resulted in the marked reduction or apparent elimination of ongoing $\beta$ cell-directed autoimmunity. Unexpectedly, the long-term reappearance of endogenous $\beta$ cell function was also observed in the pancreatic islets of the previously hyperglycemic hosts.

\section{Methods}

Animals. Female NOD mice from Taconic Farms (Germantown, New York, USA) and C57BL/6J (C57) mice from The Jackson Laboratory (Bar Harbor, Maine, USA) were maintained under pathogen-free conditions. NOD mice were screened for the onset of diabetes by monitoring body weight and blood glucose; they were diagnosed as diabetic when two consecutive blood glucose concentrations exceeded $400 \mathrm{mg} / \mathrm{dl}$. Before experimental treatments, diabetic NOD mice were maintained for 7-20 days on daily injections of 1.0-1.5 U of NPH human insulin per $100 \mathrm{~g}$ of body weight, thereby preventing immediate death and maintaining blood sugar concentration between 200 and $700 \mathrm{mg} / \mathrm{dl}$. The use of such severely diabetic mice, relatively late after disease onset, ensured that endogenous pancreatic islets were completely obliterated before the initiation of experiments. Splenocyte donors included normal C57 mice, C57 mice (C57 $\left.\beta_{2} \mathrm{M}^{-/-}\right)$in which the $\beta_{2} \mathrm{M}$ gene was disrupted, C57 mice (C57 $\beta_{2} \mathrm{M}^{-/-}$, TAP1 $\left.1^{--}\right)$in which both the $\beta_{2} \mathrm{M}$ and Tap1 genes were disrupted, and $\mathrm{MHC}$ class $\mathrm{II}^{-/-}$mice (C57 class $\left.\mathrm{II}^{-/-}\right)$in which the I-A gene was disrupted and the E locus of MHC class II was not expressed because of an endogenous defect in the C57 strain (Taconic Farms). Splenocytes $\left(9 \times 10^{6}\right)$ were injected into NOD recipients through the tail vein twice a week. CFA (Difco Laboratories, Detroit, Michigan, USA) was freshly mixed with an equal volume of physiological saline and injected $(50 \mu \mathrm{l})$ into each hind-foot pad at the time of islet transplantation or after the first splenocyte injection.

Islet transplantation. Islets were isolated from donor C57 mice or 6- to 8-week-old prediabetic female NOD mice. Density gradient centrifugation followed by hand picking of islets ensured that both preparations were highly enriched in islets and had an accurate determination of islet number. For transplantation, 500-600 freshly isolated islets were grafted beneath the left renal capsule of each diabetic NOD recipient. For islet encapsulation, 900-1,100 islets were enclosed in alginate spheres, which were then surgically inserted into the peritoneal cavity of diabetic NOD mice. Transplantation was considered successful if the nonfasting blood concentration of glucose returned to normal (<200 mg/dl) within 24 hours after surgery. The glucose concentration of orbital blood was monitored three times a week after transplantation with a Glucometer Elite instrument (Bayer Corp, Pittsburgh, Pennsylvania, USA). Body weight was also monitored three times a week. Islet grafts were considered to have been rejected if the blood glucose concentration increased to more than $250 \mathrm{mg} / \mathrm{dl}$ on two occasions. Recipients that rejected islet grafts were killed for histological examination and flow-cytometric studies. To assess the contribution of endogenous pancreatic islets to the control of blood sugar concentration, we removed subrenal islet transplants by nephrectomy. Similarly, islets encapsulated in alginate spheres, which were approximately $0.2-0.5 \mathrm{~cm}$ in diameter, were 
a

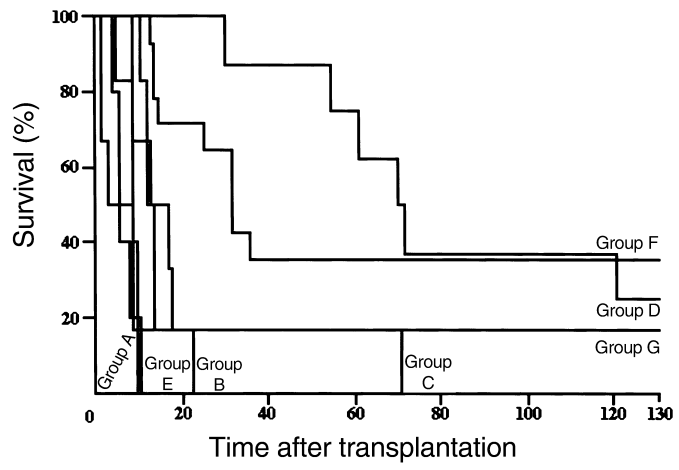

b
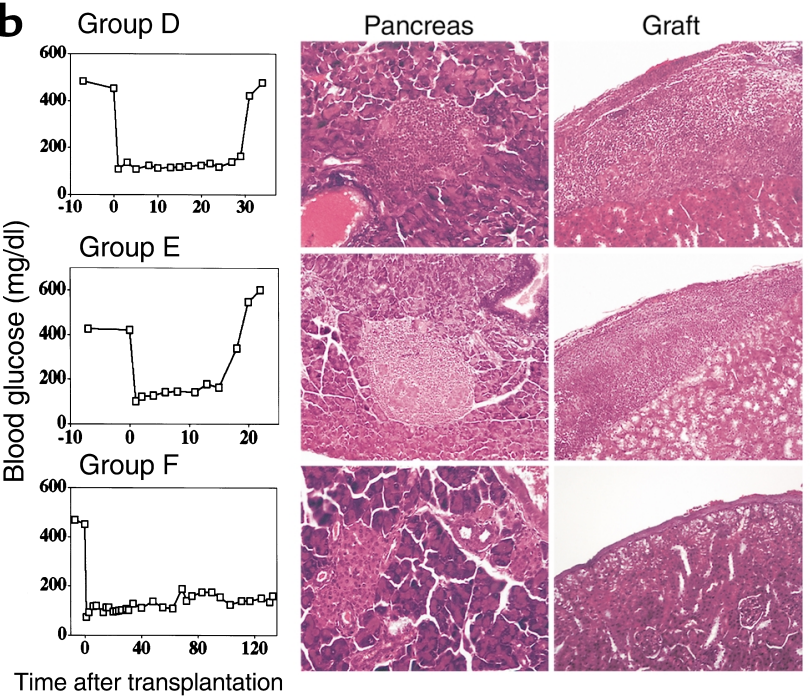

Figure 1

Kaplan-Meier cumulative survival curves (a) as well as blood glucose concentrations and histology of the pancreata and graft site (b) for diabetic NOD female mice subjected to transplantation of islets from various donor types in the absence or presence of CFA treatment. Treatment groups A-G correspond to those described in Table 1. Blood glucose concentrations were measured at the indicated times after transplantation. The histology of the pancreas and of the islet graft site under the kidney capsule was examined by staining of paraffin-embedded sections with hematoxylin and eosin. Intense lymphocyte invasion of the pancreatic islets is apparent in groups $D$ and $E$ but not in group F. $\times 400$.

removed from the peritoneal cavity by direct visualization under a dissecting microscope. Histological analysis of the pancreata and islet grafts was performed by staining with hematoxylin and eosin for evaluation of lymphocytic infiltrates and with aldehyde-fuchsin for islet insulin content. The entire pancreas from splenic to duodenal stomach attachments was removed, fixed, embedded longitudinally in a paraffin block, and subjected to serial sectioning $(10 \mu \mathrm{m})$.

Flow cytometry. Spleens were removed and gently minced on a stainless steel sieve. Cell suspensions were rendered free of red blood cells by exposure to a solution containing $0.83 \% \mathrm{NH} 4 \mathrm{Cl}$. The splenocytes were stained with mouse mAb's (PharMingen, San Diego, California, USA) to CD8 (FITC-labeled), to CD62L (phycoerythrin-labeled [PE-labeled]), to CD95 (PE- labeled), or to CD45RB (PE-labeled), and were analyzed (>10,000 cells per sample) the same day with an Epics Elite flow cytometer. Spleen cells were incubated for 24 hours in the absence or presence of TNF- $\alpha(20 \mathrm{ng} / \mathrm{ml})$, after which apoptotic cells were detected by flow cytometry with FITC-conjugated annexin V. Apoptotic cells were defined as cells positive for both propidium iodide (PI) and annexin V staining; numbers within the upper quadrants represent the corresponding percentages of cells.

Adoptive transfer. Adoptive transfer was performed as described (29). Recipient male NOD mice, 4-8 weeks of age, were irradiated (790 rads) with a ${ }^{137} \mathrm{Cs}$ source and injected intravenously within 2 hours of irradiation with donor splenocytes $\left(2 \times 10^{7}\right.$ viable cells) suspended in $0.25 \mathrm{ml}$ of serum-free medium. Diabetic spleen cell donors were female NOD mice that typically had exhibited blood sugar concentrations of greater than 400 $\mathrm{mg} / \mathrm{dl}$ for at least 3 weeks. Multiple diabetic donor spleens were pooled to produce sufficient cells for all of the hosts in a given experiment.

Statistics. Exact algorithm $P$ values were calculated in some instances with multiple comparisons corrected by the number of tested variables. A $P$ value less than 0.05 was considered statistically significant.

\section{Results}

CFA treatment and islet transplantation in NOD mice. Hosts for the transplantation experiments were severely diabetic female NOD mice, usually more than 20 weeks of age, that had exhibited blood glucose concentrations of greater than $400 \mathrm{mg} / \mathrm{dl}$ for at least 7 days and had been treated by daily administration of insulin to prevent death. Islet transplants were placed unilaterally under the kidney capsule to facilitate nonlethal removal and histological examination. Islets from 6- to 8-week-old prediabetic NOD females (recipient group A) or from normal C57 mice (recipient group B) were rapidly rejected by diabetic NOD recipients (Table 1 , Figure 1a). Although C57 donor islets with transient ablation of MHC class I expression survive indefinitely

\section{Table 1}

Duration of normoglycemia in diabetic NOD mice treated by intrarenal transplantation of islets from various donor types, with or without CFA

\begin{tabular}{|c|c|c|c|}
\hline Group & Donor & CFA & $\begin{array}{l}\text { Individual values for duration } \\
\text { of normoglycemia (days) }\end{array}$ \\
\hline A & NOD & $(-)$ & $4,6,6,8,10$ \\
\hline B & C57 & $(-)$ & $2,2,3,9,9,23$ \\
\hline C & $\mathrm{C} 57 \beta_{2} \mathrm{M}^{-/-}$ & $(-)$ & $5,9,12,17,18,71$ \\
\hline D & NOD & $(+)$ & $30,55,61,70,72,121,136,>137$ \\
\hline $\mathrm{E}$ & C57 & $(+)$ & $9,9,9,10,11$ \\
\hline $\mathrm{F}$ & $\mathrm{C} 57 \beta_{2} \mathrm{M}^{-/-}$ & $(+)$ & $\begin{array}{c}13,14,14,15,25,32,32,32,36 \\
>129,>131,>132,>133,>133\end{array}$ \\
\hline $\mathrm{C} 5$ & $7 \beta_{2} \mathrm{M}^{-/-}$, TAP1-/- & $(+)$ & $11,12,13,14,14,>148$ \\
\hline
\end{tabular}

$P=0.0083$, group $\mathrm{F}$ versus all other groups (Wilcoxon test); $P=0.00017$, group $\mathrm{F}$ versus group $\mathrm{A} ; P=0.0036$, group $\mathrm{F}$ versus group $\mathrm{B} ; P=0.0375$, group F versus group $C ; P=0.33$, group $F$ versus group $D ; P=0.0002$, group $F$ versus group $\mathrm{E} ; P=0.03$, group $\mathrm{F}$ versus group $\mathrm{G}$ (Wilcoxon test). 


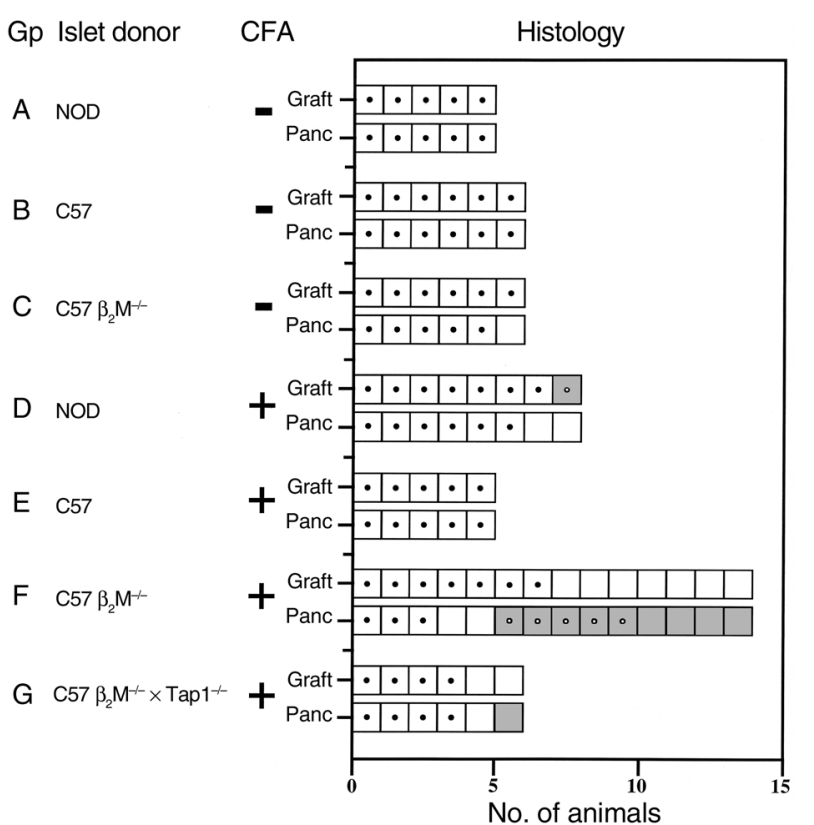

Figure 2

Summary of the histological characteristics of the graft site and pancreas of individual NOD hosts subjected to transplantation of islets from various donor types in the absence or presence of CFA treatment. Animals correspond to those listed in Table 1. Open squares, absence of visible islet structures and of lymphocytic accumulation; open squares with center dots, massive lymphocytic accumulation invading or obscuring islet remnants; shaded squares, viable islets without lymphocytes; shaded squares with center dots, viable islet structures with only circumferential lymphocytic accumulation. Examination of serial longitudinal sections of the entire pancreas revealed the presence of approximately $25-35$ islets in control BALB/c mice and approximately 12-20 islets in successfully treated NOD mice (group F). Gp, group; Panc, pancreas. $P<0.0001$, group F versus all other groups; $P<0.007$, group $\mathrm{F}$ versus group $\mathrm{D}$ (Wilcoxon test).

in nonautoimmune diabetic hosts (6), the survival time of islets from $\beta_{2} \mathrm{M}^{-/-} \mathrm{C} 57$ mice in diabetic NOD females (group C) was only about three times that of normal C57 islets. As expected from previous observations (2, 32 ), treatment with CFA prolonged the survival of syngeneic islet grafts in diabetic NOD hosts (group D) but had a minimal effect on the survival of C57 islets (group E), which were uniformly rejected by 11 days after transplantation. However, the combination of $\beta_{2} M^{-/-}$C57 islet transplants with CFA treatment resulted in sustained (>129 days) normoglycemia in 5 of 14 diabetic NOD hosts (group F). Although the duration of hyperglycemia before initiation of therapy varied between 7 and 20 days, the length of this interval was not significantly related to the duration of sustained normoglycemia after treatment (data not shown). The animals that exhibited sustained normoglycemia also demonstrated progressive weight gain, similar to that apparent in NOD female cohorts who never became diabetic (data not shown). With normalization of blood sugar concentration as a measure of treatment success, the success of $\beta_{2} \mathrm{M}^{-/-}$C57 islet transplantation together with CFA administration was significantly different from that apparent with the other groups combined but did not differ from that of NOD islet transplantation together with CFA treatment.

After the recurrence of hyperglycemia in the NOD mice that had been treated with CFA and syngeneic (NOD) islet transplants, the kidney containing the islet graft was examined histologically. Marked lymphocytic infiltration was apparent under the kidney capsule at the site of transplantation, a characteristic of recurrent autoimmune disease (Figure 1b, group D); moreover, no intact islets were detected in the pancreas, although islet remnants, largely obscured by dense pockets of infiltrating lymphocytes, were evident. Similar histological characteristics of both the transplant site and pancreas were apparent in diabetic NOD mice that had received CFA and islet grafts from C57 donors (Figure 1b, group E). Unexpectedly, for all five NOD mice with long-term normoglycemia after receiving
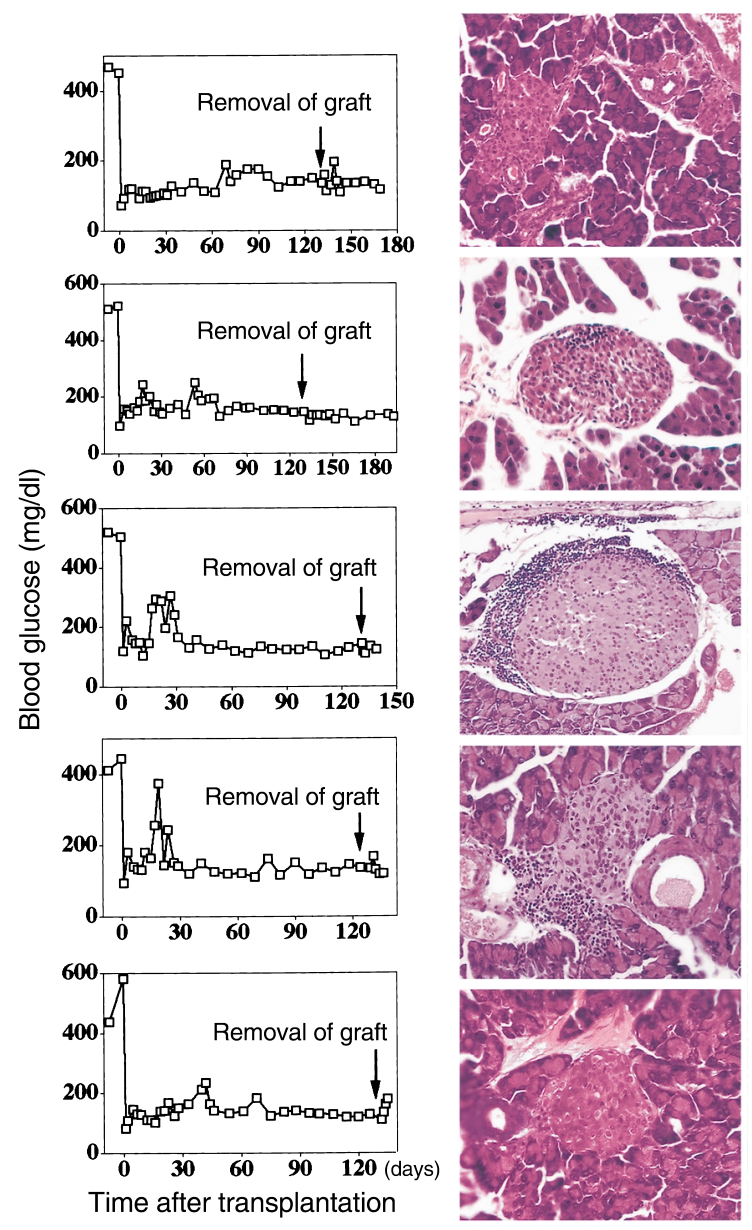

\section{Figure 3}

Blood glucose concentrations (left panels) and histology of the pancreata (right panels) of diabetic NOD female mice treated with CFA and intrarenal transplantation of islets from $\beta_{2} \mathrm{M}^{-/-} \mathrm{C} 57$ mice. The kidneys containing the islet grafts were removed at the times after transplantation indicated by the arrows. The animals were killed at various times thereafter, and the pancreata were subjected to histological staining with hematoxylin and eosin. Representative islets are shown. $\times 400$. 
$\beta_{2} M^{-/-}$C57 islets and CFA treatment, no surviving islet grafts were detected under the kidney capsule when the animals were killed at more than 129 days after transplantation (Figure 1b, group F). In contrast, the pancreas of each of these five recipients exhibited wellformed islets that appeared completely granulated when stained by aldehyde-fuchsin. The islets were free of lymphocytes or lymphocytes were present only circumferentially; this latter pattern of lymphocyte accumulation, with lymphocytes surrounding but not invading the islets, has been associated with nonprogressive or interrupted $\beta$ cell autoimmunity (33). The return to normoglycemia in the absence of detectable transplanted islet tissue, together with the presence of islets in a pancreas largely devoid of infiltrating lymphocytes, suggested not only that autoimmunity had been interrupted but that the function of endogenous $\beta$ cells had been restored.

Restoration of near-normal pancreatic islet histology was observed only in the diabetic NOD mice that received both $\beta_{2} \mathrm{M}^{-/-}$islet grafts and CFA treatment (Figure 2). Pancreatic islets were thus not detected in any diabetic NOD mice treated with CFA and syngeneic NOD islets; the persistence of normoglycemia in such recipients appeared solely due to the transplanted islets, which always exhibited invasive insulitis (Figure 1b, Figure 2). Thus, treatment with CFA together with syngeneic NOD islets may have slowed disease recurrence, but persistent autoimmunity remained.

The relative contributions of restored endogenous pancreatic islets and transplanted islets to the maintenance of normoglycemia in NOD mice treated with CFA and islet grafts from $\beta_{2} M^{-/-}$C57 donors were assessed by removal of the kidney containing the islet transplant after 120 days of normoglycemia in a second group of five animals. All five mice remained normoglycemic after nephrectomy until they were killed 3-60 days later (Figure 3). Histological analysis of the kidneys that received the grafts revealed a complete loss of identifiable islet structures. In contrast, the pancreata of all five recipients contained well-formed islets either without lymphocytic infiltration or with circumferen- tially distributed lymphocytes only. Normoglycemia after nephrectomy was thus maintained solely by endogenous pancreatic islets. In contrast, nephrectomies performed during the posttransplantation period of normoglycemia (day 62, day 85) in two mice who had received CFA plus syngeneic NOD islets resulted in a rapid return to hyperglycemia (data not shown), demonstrating that the control of blood sugar in this treatment group was mediated solely by the transplanted islet tissue.

We also transplanted diabetic NOD females with islets from C57 mice in which the genes for both $\beta_{2} \mathrm{M}$ and TAP1 had been deleted. Together with TAP2, TAP1 mediates the transport of endogenous peptides from the cytosol into the lumen of the endoplasmic reticulum for their assembly with MHC class I molecules (34). Islet cells from these mice are more permanently defective in presentation of self antigens by MHC class I than are those from $\beta_{2} \mathrm{M}^{-/-}$mice. Transplantation of $\beta_{2} \mathrm{M}^{-/-}$, TAP1 $1^{-/-}$C57 islets combined with injection of CFA resulted in the return of hyperglycemia within 14 days in five of six animals (group G); histological examination of the pancreata revealed a pattern typical of that for untreated diabetic NOD mice (Table 1, Figure 2). Thus, a transient interruption of peptide presentation by donor MHC class I molecules is essential for the abrogation of autoimmunity, whereas a sustained interruption of this process prevents the reestablishment of tolerance and the restoration of endogenous pancreatic islet integrity.

CFA treatment and splenocyte injection in NOD mice. Given that the restoration of normoglycemia in the diabetic NOD mice treated with CFA and $\beta_{2} M^{-/-}$C57 islets did not depend on the continuing secretion of insulin by the islet grafts, we next investigated whether C57 donor cell types other than islets might serve a similar therapeutic role. Nine diabetic NOD mice were treated with a single bilateral injection of CFA followed by a 40-day regimen of biweekly intravenous injections of C57 splenocytes. These lymphoid cells express both MHC class I and class II proteins and survive only transiently in NOD hosts because of graft rejection (data not shown). Repeat injec-

\section{Figure 4}

Effect of CFA treatment, repeated infusions of C57 splenocytes, and daily insulin injections on glycemic control and islet morphology in diabetic NOD recipients. Blood glucose concentration was monitored for up to 40 days in diabetic NOD females that were treated either with daily injections of insulin alone (controls, $n=4$ ) (a) or with a single bilateral injection of CFA, daily insulin injections (until normoglycemia was restored), and biweekly injections of C57 splenocytes $(n=9)$ (b). Pancreatic histology was examined by hematoxylin and eosin staining either at the time of death (arrows) of control animals (c) or after the 40-day observation period for animals receiving the experimental treatment; of the latter mice, representative islets are shown for one of the four animals that remained hyperglycemic (d) and for one of the three animals in which normoglycemia was restored $(\mathbf{e}) . \times 400$.

\section{a}
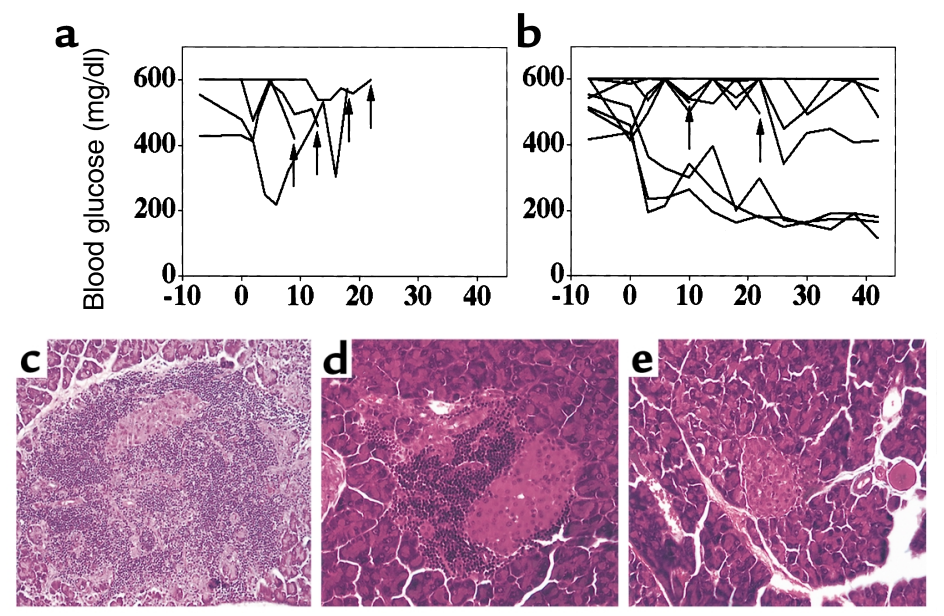


\section{Table 2}

Impact of short-term control of blood sugar on rescue of endogenous islets in diabetic NOD mice

\begin{tabular}{|c|c|c|c|c|}
\hline Group & Spleen cell donor & CFA & $\begin{array}{l}\text { Normoglycemic/ } \\
\text { total recipients }\end{array}$ & Percentage \\
\hline$A$ & & $(-)$ & $0 / 7$ & 0 \\
\hline B & & $(+)$ & $0 / 6$ & 0 \\
\hline C & $\mathrm{C} 57$ & $(+)$ & $7 / 9$ & 78 \\
\hline $\mathrm{D}$ & C57 $\beta_{2} M^{-/-}$, TAP1-/- & $(+)$ & $2 / 6$ & 33 \\
\hline $\mathrm{E}$ & C57 class $\mathrm{II}^{-/-}$ & $(+)$ & $8 / 11$ & 73 \\
\hline $\mathrm{F}$ & C57 + anti-TNF- $\alpha$ & $(+)$ & $0 / 5$ & 0 \\
\hline
\end{tabular}

Diabetic NOD females were implanted intraperitoneally with C57 islets encapsulated in alginate spheres. They then received no further treatment (group $A$ ), a single bilateral injection of CFA only (group B), or CFA treatment plus biweekly intravenous injections of splenocytes from normal $C 57$ mice (groups $C$ and $\mathrm{F}$ ), $\beta_{2} \mathrm{M}^{-/-}$, TAP1-/- C57 mice (group D), or $\mathrm{MHC}$ class $11^{-/-} \mathrm{C} 57$ mice (group E). Animals in group $F$ also received daily injections of a mAb to TNF- $\alpha$ for the first 10 days of treatment. Encapsulated islets were removed from all animals after approximately 40 days. $P=0.000069$, groups $C$ and $E$ versus all other groups. Despite the fact that many comparisons were made, the $P$ value remained less than 0.05 when multiplied by the different comparisons between groups.

tions of splenocytes ensure that the host is continuously exposed to intact antigen presentation complexes on the surface of these cells. The recipients were monitored for hyperglycemia every 3 or 4 days, and insulin was administered daily unless normoglycemia returned. A control group of four diabetic NOD mice received daily insulin injections only. All four control mice died on or before day 25 of the experimental period as a result of poor control of blood glucose and consequent ketosis and cachexia (Figure 4a). In contrast, seven of the nine mice injected with CFA and C57 splenocytes were alive after 40 days, and three of these animals had become normoglycemic and insulin independent (Figure 4b). The pancreata of control (insulin treatment only) mice exhibited pronounced lymphocytic infiltrates that obscured any remaining islet structures (Figure 4c). The pancreata of the four NOD mice treated with CFA and C57 splenocytes that remained alive but hyperglycemic and insulin dependent revealed a marked decrease (relative to control animals) in the number of lymphoid infiltrates, which were located either circumferentially or adjacent to the infrequent islet structures (Figure 4d). On killing of each of the three NOD mice treated with CFA and C57 splenocytes that maintained normoglycemia after discontinuation of insulin injections, the pancreata exhibited abundant islets that were free of invasive lymphocytes or were associated only with circumferential lymphocytes (Figure 4e). Thus, treatment with CFA combined with repeated exposure to C57 lymphocytes resulted in complete reversal of diabetes in approximately $30 \%$ of NOD recipients and partially restored $\beta$ cell function in an additional approximately $40 \%$ of recipients.

Influence of glycemic control on restoration of endogenous islet function. The reversal of diabetes in NOD mice by CFA and repeated exposure to $\mathrm{C} 57$ splenocytes indicated that restoration of endogenous islet function is achievable without islet transplantation and despite the poor glycemic control attained by insulin injections. The ben- eficial influence of glycemic control on the growth, survival, and function of cultured islets, as well as of transplanted islets in nonautoimmune settings, has been demonstrated $(35,36)$. To determine whether the restoration of endogenous $\beta$ cell function could be achieved more consistently with better control of blood glucose, we replaced insulin injections with the intraperitoneal implantation of alginate-encapsulated C57 mouse islets. Alginate encapsulation prevents direct contact between donor endocrine cells and host $T$ cells, and such grafts have been shown to provide near-normal glycemic control for 40 to 50 days in approximately $70-80 \%$ of autoimmune NOD recipients (37).

Almost all diabetic NOD mice that received alginateencapsulated C57 islets exhibited improved glucose regulation or normoglycemia. The alginate spheres were removed 40-50 days after implantation, and blood glucose concentration was monitored (Table 2). The seven mice treated only with alginate-encapsulated islets (group A), the six mice that received a single bilateral injection of CFA (group B), and the three mice treated with biweekly injections of C57 splenocytes (data not shown) all exhibited a rapid return to hyperglycemia and early death after removal of the implants (Table 2, Figure 5). The pancreata of NOD mice that received only alginate-encapsulated islets revealed no sign of intact islets or of lymphoid infiltrates (data not shown). The pancreata of NOD hosts treated with CFA and alginate-encapsulated islets exhibited marked invasive insulitis that obscured islet structures (Figure 6). In contrast, seven of the nine (78\%) diabetic NOD mice that received CFA and C57 splenocytes (group C) remained normoglycemic for more than 40 days (until killing) after removal of the alginate-encapsulated islets (Figure 5, Table 2). The pancreata of these animals contained large islets with circumferentially distributed lymphocytes (Figure 6). The islet mass after at least 80 days of disease reversal was estimated at approximately $50 \%$ of the original value. The pancreata from con-

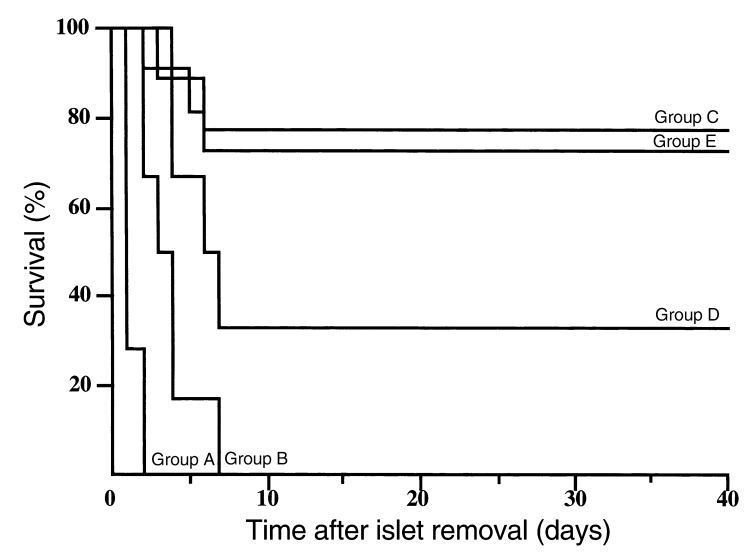

Figure 5

Kaplan-Meier cumulative survival of diabetic NOD mice after removal of alginate-encapsulated islets. Treatment groups A-E correspond to those described in Table 2. 
trol BALB/c mice contained approximately 25-35 islets; the pancreata from successfully treated NOD mice contained approximately $12-20$ islets with serial histological sections. Thus, maintenance of normoglycemia during the treatment period increased the percentage of diabetic mice cured of hyperglycemia.

Role of TNF- $\alpha$ in treatment outcome. We attempted to identify features of the successful treatment regimens that are critical to a positive outcome. We had used CFA to induce the endogenous production of TNF- $\alpha$ (31). The importance of TNF- $\alpha$ in treatment success was therefore investigated by the intravenous administration of a rat IgG1 mAb to this cytokine (clone MP6X73; Accurate Chemical \& Scientific Corp., Westbury, New York, USA) at a dose of $1.5 \mathrm{mg} /$ day for the first 10 days in diabetic NOD hosts treated with C57 splenocytes, CFA, and alginate-encapsulated islets. All five NOD mice so treated exhibited a rapid return to hyperglycemia on removal of the alginate-encapsulated islets 50-70 days after transplantation (Table 2 , group F), consistent with the notion that TNF- $\alpha$ plays an obligatory role in the beneficial effect of CFA. This effect of the $m A b$ to TNF- $\alpha$ was specific, given that administration of a rat IgG1 mAb to the human $T$ cell receptor $V_{\beta} 1$ chain (clone BL37.2; American Type Culture Collection, Rockville, Maryland, USA) at a dose of $1.5 \mathrm{mg} /$ day for 10 days did not affect the success of treatment with C57 splenocytes and CFA (data not shown). Direct administration of TNF- $\alpha$ to diabetic hosts was not feasible because of the prohibitive cost.

We next investigated whether the production of TNF- $\alpha$ in diabetic NOD mice treated with CFA results in the selective elimination of autoreactive lymphoid cells first by examining the susceptibility of lymphocytes from successfully treated animals to TNF- $\alpha$-induced cell death in vitro. As shown previously $(26,27)$, incubation of normal C57 spleen cells with TNF- $\alpha$ in vitro had no effect on cell viability; for the animal shown in Figure 7a, the proportion of apoptotic cells was $0.01 \%$ for splenocytes incubated in the absence or presence of TNF- $\alpha$. In contrast, exposure of splenocytes from an untreated 20-week-old NOD female to TNF- $\alpha$ in vitro increased the proportion of apoptotic cells from 0.03 to $38.3 \%$. Such an increased sensitivity to TNF$\alpha$-induced apoptosis in vitro was no longer evident with spleen cells derived from NOD mice cured of diabetes; thus, splenocytes from a NOD female successfully treated with both CFA and C57 splenocytes (Table 2, group C) exhibited 23.2 and $23.9 \%$ apoptosis in the absence and presence of TNF- $\alpha$, respectively (Figure 7a). Successful therapy generated a subpopulation of nonpathologic but TNF$\alpha$-resistant T cells that exhibited an increased tendency to undergo cell death in culture (Figure 7a). Disease reversal, even 210 days after cessation of treatment, was thus associated with the persistent elimination of TNF- $\alpha$-sensitive T cells, a popula- tion of cells with a demonstrated ability to play a role in disease $(29,30)$. The permanent elimination of these formerly abundant TNF- $\alpha$-sensitive lymphoid cells, presumably in response to TNF- $\alpha$ (and, perhaps, to other CFA-induced cytokines), was observed uniformly in successfully treated diabetic NOD mice. Similar complete and stable elimination of TNF- $\alpha$-sensitive cells at various times after treatment has been observed in more than 20 NOD mice.

We also examined the effect of TNF- $\alpha$ on the pathogenesis of autoimmune diabetes in adoptive transfer experiments. Young recipient NOD males were subjected to irradiation followed by an intravenous injection of donor splenocytes either from newly diabetic NOD females or from NOD mice with long-term normoglycemia due to treatment with CFA and C57

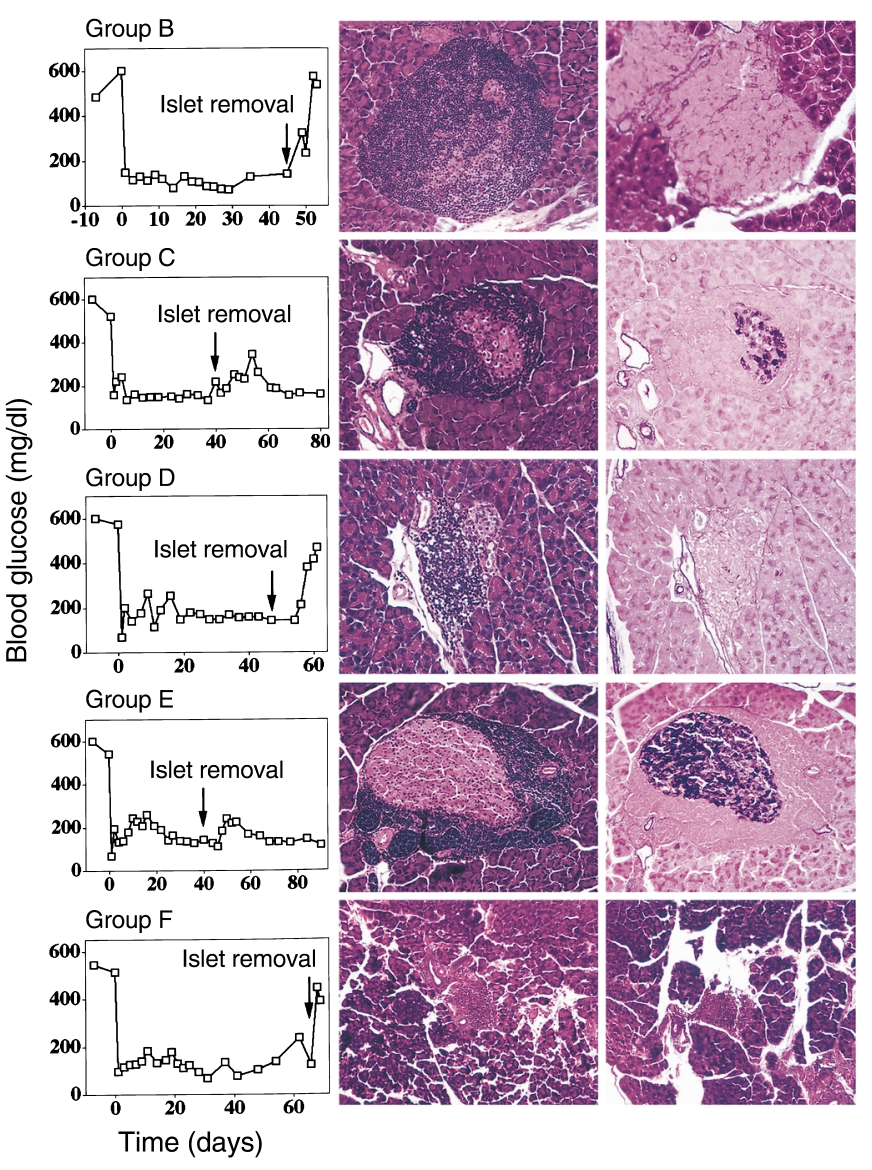

Figure 6

Blood glucose control and pancreatic islet histology in diabetic NOD mice treated with alginate-encapsulated islets, CFA, and splenocytes from various donor types. Diabetic NOD females were treated as described in Table 2, and data from representative animals of groups $B$ through $F$ are shown. Blood glucose concentration was monitored before and after the removal (arrows) of the alginate-encapsulated islets (left panels). The histology of the pancreas was analyzed at the time of killing, indicated by the last blood glucose measurement; sections were stained with hematoxylin and eosin (middle panels) and/or with aldehyde-fuchsin (right panels). Lymphocytic invasion of islets is apparent in the animals of groups $B, D$, and $F$, whereas lymphocytic infiltrates surround the islets in the animals of groups $C$ and E. $\times 400$. 
$\mathbf{a}$

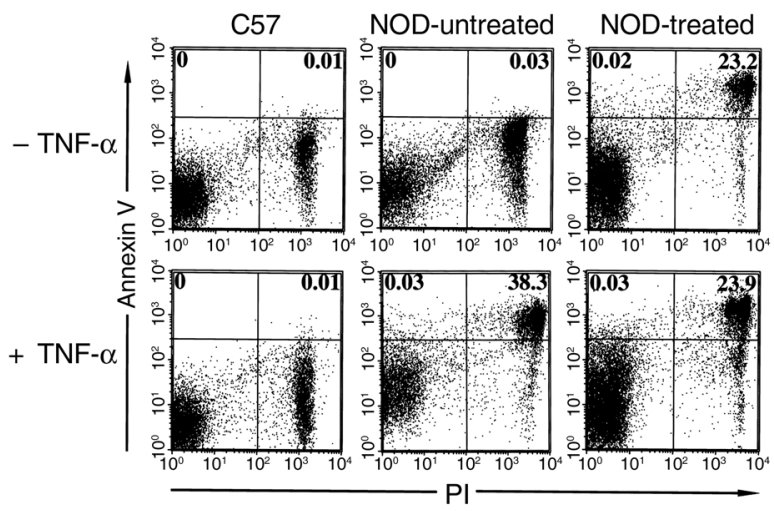

b

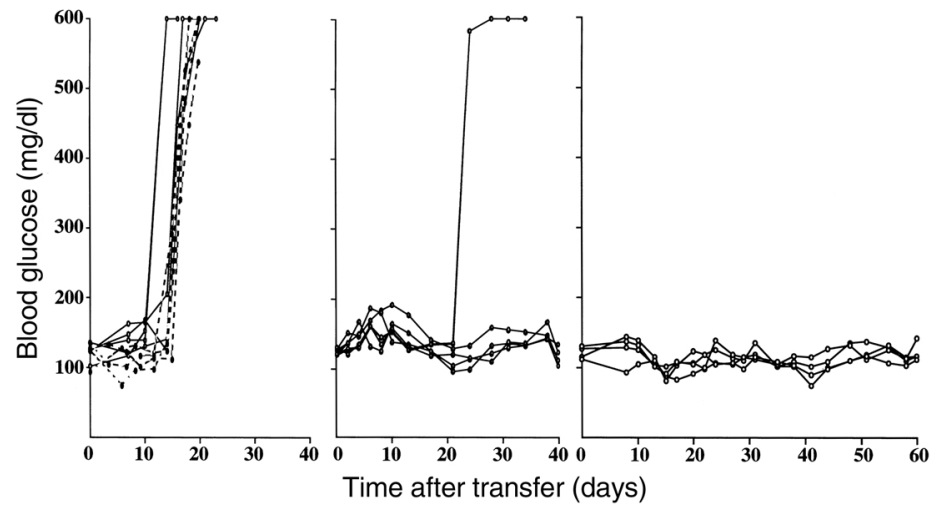

C
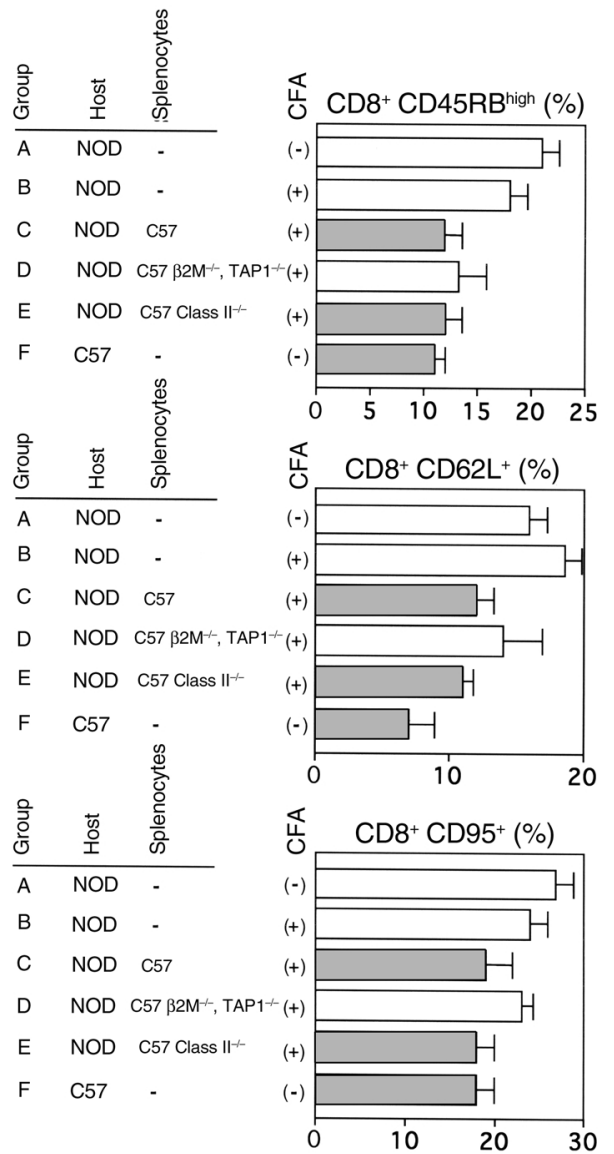

Figure 7

Roles of TNF- $\alpha$ and MHC class I peptide complexes in reversal of diabetes in NOD mice. (a) Effect of TNF- $\alpha$ on the survival of spleen cells derived from a control C57 mouse or from untreated or successfully treated NOD female mice. (b) Effect of TNF- $\alpha$ treatment of splenocytes from diabetic NOD mice on the adoptive transfer of disease and the inability of splenocytes from successfully treated NOD mice to transfer disease. Young male NOD mice were irradiated and then injected with diabetic NOD female splenocytes either immediately after their isolation (left panel, dashed lines) or after incubation for 24 hours in the absence (left panel, solid lines) or presence (middle panel) of TNF- $\alpha$ $(20 \mathrm{ng} / \mathrm{ml})$; alternatively, four irradiated hosts each received splenocytes from a different NOD donor with long-term normoglycemia restored by CFA and C57 spleen cell injections (right panel). (c) Flow cytometric analysis of the percentages of CD8 ${ }^{+} \mathrm{CD} 45 \mathrm{RB} \mathrm{B}^{\text {high }}, \mathrm{CD} 8^{+} \mathrm{CD} 62 \mathrm{~L}^{+}$, and $\mathrm{CD} 8{ }^{+} \mathrm{CD} 95^{+}$cells among splenocytes of mice from various treatment groups. Diabetic NOD females were implanted intraperitoneally with alginate-encapsulated C57 islets. They then received no further treatment (group A), a single bilateral injection of CFA only (group B), or CFA treatment plus biweekly intravenous injections of splenocytes from normal C57 mice (group C), $\beta_{2} M^{-{ }^{-}}$, TAP1 $1^{-/-}$C57 mice (group D), or $\mathrm{MHC}$ class $\mathrm{II}^{-/-} \mathrm{C} 57$ mice (group E). Shaded bars represent C57 control mice (group F) or NOD mice that exhibited normoglycemia and disease reversal after removal of alginate-encapsulated islets (groups $\mathrm{C}$ and $\mathrm{E}$ ); open bars represent untreated NOD mice (group A) or NOD mice subjected to treatments that did not result in disease reversal (groups B and D).

splenocytes. The onset of diabetes was observed in all recipients by day 15 after the transfer of diabetic mouse cells that were injected either immediately after isolation or after control culture for 24 hours (Figure 7b, left panel). In contrast, four of the five recipients of diabetic mouse cells that had been cultured with TNF- $\alpha$ for 24 hours before adoptive transfer remained normoglycemic for at least 40 days after cell injection (Figure $7 \mathrm{~b}$, middle panel). Furthermore, the four NOD hosts each injected with splenocytes from a different NOD female that had experienced reversal of autoimmunity for more than 120 days failed to develop disease during observation periods of more than 60 days (Figure $7 \mathrm{~b}$, right panel). TNF- $\alpha$-resistant NOD splenocytes, enriched either in vitro by direct exposure of cells to TNF- $\alpha$ or in vivo by disease reversal, are thus incapable of transferring disease to naive hosts.

Role of MHC class I peptide complexes in T cell selection and treatment outcome. Disease reversal in diabetic NOD mice required treatment with both CFA and cells that express MHC class I peptide complexes. Only two of six (33\%) diabetic NOD mice that received CFA and biweekly injections of splenocytes from $\beta_{2} \mathrm{M}^{-/-}, \mathrm{TAP1}^{-/-} \mathrm{C} 57$ donors remained normoglycemic after removal of alginate-encapsulated islets (Table 2, group D). The pancreata of the four animals that became hyperglycemic after removal of the alginate spheres contained no granulated islets and only a few visible islet structures, which were invaded and obscured by lymphocytic infiltrates (Figure 6). In contrast, 8 of 11 (73\%) diabetic NOD mice 
treated with CFA and splenocytes from C57 donors lacking MHC class II protein expression remained normoglycemic after removal of the alginate-encapsulated islets (Table 2, group E); the pancreata of these animals contained large islets that exhibited only moderate lymphocytic accumulation at the periphery (Figure 6).

Interruption of antigen presentation by MHC class I skews the $\mathrm{T}$ cell repertoire to an overabundance of naive cells, a consistent trait of diabetes-prone NOD mice and humans (38-40). Improper $\mathrm{T}$ cell selection secondary to interruption of antigen presentation by MHC class I results in overexpression of CD95 by CD8 ${ }^{+} \mathrm{T}$ cells as well as an increase in the abundance of cells with naive cell markers such as $\mathrm{CD} 62 \mathrm{~L}^{+}$and $\mathrm{CD} 45 \mathrm{RB}$ high $(12,23)$. To investigate whether therapeutic reversal of NOD mouse diabetes was associated with a change in naive $T$ cell selection, we subjected splenocytes to flow-cytometric analysis. Flow cytometry was performed 5 to 26 days after removal of the alginate-encapsulated islets and termination of therapy.

Untreated NOD mice exhibited the expected increases in the abundance of naive $\mathrm{CD}^{+} \mathrm{CD} 45 \mathrm{RB}^{\text {high }}, \mathrm{CD}^{+}{ }^{+} \mathrm{CD} 62 \mathrm{~L}^{+}$, and $\mathrm{CD}^{+} \mathrm{CD}^{+} 5^{+}$cells compared with $\mathrm{C} 57$ animals (Figure $7 \mathrm{c}$ ). In contrast, in NOD female mice that were successfully treated with alginate-encapsulated islets, CFA, and administration of C57 splenocytes, the percentages of each of these cell populations were reduced to normal or nearnormal values. The abnormally high numbers of $\mathrm{CD}^{+} \mathrm{CD} 45 \mathrm{RB}^{\text {high }}, \mathrm{CD}^{+} \mathrm{CD}^{2} 2 \mathrm{~L}^{+}$, and $\mathrm{CD}^{+}{ }^{+} \mathrm{CD} 95^{+}$cells remained increased in diabetic NOD females treated with alginate-encapsulated islets and CFA, either alone or together with administration of $\beta_{2} \mathrm{M}^{-/-}$, TAP1-/- C57 splenocytes. Data are means plus or minus SEM of values from at least five mice per group. Exact algorithm $P$ values were calculated for comparisons of each cell population between groups $\mathrm{C}, \mathrm{E}$, and $\mathrm{F}$ versus groups $\mathrm{A}, \mathrm{B}$, and $\mathrm{D}: P=0.001$ for CD8 ${ }^{+} \mathrm{CD} 45 \mathrm{RB}^{\text {high }}$ cells, $P=0.01$ for $\mathrm{CD} 8{ }^{+} \mathrm{CD} 62 \mathrm{~L}^{+}$cells, and $P=0.05$ for $\mathrm{CD}^{+} \mathrm{CD}^{+} 5^{+}$cells. Despite the fact that many comparisons were performed, the $P$ value remained less than 0.05 even when multiplied by the three comparisons. These data showed that the $\mathrm{T}$ cell reselection apparent in successfully treated NOD mice was secondary to reexposure to complexes of MHC class I molecules and self peptides. The normalization of $\mathrm{T}$ cell phenotype did not require reexposure to MHC class II-peptide complexes, given that the administration together with CFA and alginate-encapsulated islets of $\mathrm{MHC}$ class $\mathrm{II}^{-/}$splenocytes was as effective as was that of normal C57 splenocytes.

\section{Discussion}

We have demonstrated the effectiveness of a novel therapy for the correction of established autoimmune diabetes in the NOD mouse. Three aspects of this treatment regimen appear to operate in parallel and in a synergistic manner: (a) Injection of CFA, and the consequent induction of TNF- $\alpha$, results in the elimination of TNF- $\alpha$-sensitive cells, which have been shown previously to transfer existing disease (28-31); (b) the introduction of functional MHC class I peptide complexes expressed on the surface of either normal islet cells or normal lymphocytes results in partial but stable reselection of the
$\mathrm{T}$ cell population of the NOD host, leading to an increase in the abundance of long-term memory $\mathrm{T}$ cells $(6,12,23)$; and (c) suppression of hyperglycemia, although not obligatory, promotes the functional restoration of endogenous $\beta$ cells or their precursors.

We propose that continuous or repeated exposure to parenchymal or lymphoid cells expressing MHC class I molecules and self peptides initiates the reeducation of host $\mathrm{T}$ cells, which was apparent in CFA-treated hosts from the loss of cells with an increased sensitivity to TNF- $\alpha$-induced apoptosis and from the restoration of a cell surface phenotype characteristic of long-term memory cells. This reeducation resulted in the establishment of long-term tolerance, as demonstrated by the elimination of both recurrent hyperglycemia and invasive insulitis. Treatment of NOD mice with severe hyperglycemia and islet destruction resulted in the reappearance of pancreatic insulin-secreting cells and normoglycemia. The rate of pancreatic $\beta$ cell proliferation is increased during the active phase of disease in NOD mice, and NOD islet stem cells proliferate in culture (41). The interruption of $\beta$ cell autoimmunity may promote both the rescue of surviving $\beta$ cells in islets as well as the production of new $\beta$ cells that are now able to survive in the altered immunological milieu. The expression of MHC class I molecules and self peptides by NOD pancreatic $\beta$ cells (21) may be responsible for maintenance of peripheral tolerance after termination of disease by transient therapy.

The application of this therapy to humans with type 1 diabetes may be feasible. As in NOD mice, lymphocytes from type 1 diabetic humans show an increased sensitivity to TNF- $\alpha$-induced apoptosis (10) as well as age-related defects in MHC class I presentation of self peptides for proper $T$ cell selection $(11,13)$. Moreover, diabetic humans continue to produce auto-Ab's to islet targets for several years after the onset of frank hyperglycemia, indicating the persistence of islet cell antigen expression. Thus, a proportion of individuals with type 1 diabetes may possess a $\beta$ cell mass or islet regenerative potential similar to that of hyperglycemic NOD mice. Even if the regenerative capacity of $\beta$ cells is exhausted, a similar immunomodulation approach may provide a less hostile milieu for islet replacement.

\section{Acknowledgments}

This work was supported by The Iacocca Foundation. We thank Biohybrid Technologies (J. Hayes, D. Wolf, and C. McGrath) for assistance with islet preparation and encapsulation; S. Thompson (Bayer Corp.) for providing surplus blood glucose monitoring strips; $\mathrm{M}$. Contant for preparation of specimens for histological analysis; NICHD for funding to study autoimmune PDF patients; and J. Avruch and D. Nathan for critical review of the manuscript.

1. Nomikos, I.N., Prowse, S.J., Carotenuto, P., and Lafferty, K.J. 1986. Combined treatment with nicotinamide and desferrioxamine prevents islet allograft destruction in NOD mice. Diabetes. 35:1302-1304.

2. Wang, T., Singh, B., Warnock, G.L., and Rajotte, R.V. 1992. Prevention of recurrence of IDDM in islet-transplanted diabetic NOD mice by adju- 
vant immunotherapy. Diabetes. 41:114-117.

3. Lakey, J.R., Singh, B., Warnock, G.L., and Rajotte, R.V. 1994. BCG immunotherapy prevents recurrence of diabetes in islet grafts transplanted into spontaneously diabetic NOD mice. Transplantation. 57:1213-1217.

4. Chatenoud, L., Primo, J., and Bach, J.F. 1997. CD3 antibody-induced dominant self tolerance in overtly diabetic NOD mice. J. Immunol. 158:2947-2954.

5. Shapiro, A.M., et al. 2000. Islet transplantation in seven patients with type 1 diabetes mellitus using a glucocorticoid-free immunosuppressive regimen. N. Engl. J. Med. 343:230-238.

6. Faustman, D., and Coe, C. 1991. Prevention of xenograft rejection by masking donor HLA class I antigens. Science. 252:1700-1702.

7. Markmann, J.F., et al. 1992. Indefinite survival of MHC class I-deficient murine pancreatic islet allografts. Transplantation. 54:1085-1089.

8. Osorio, R.W., Asher, N.L., Melzer, J.S., and Stock, P.G. 1994. Beta-2microglobulin gene disruption prolongs murine islet allograft survival in NOD mice. Transplant. Proc. 26:752.

9. Yan, G., Fu, Y., and Faustman, D.L. 1997. Reduced expression of Tap1 and Lmp2 antigen processing genes in the nonobese diabetic (NOD) mouse due to a mutation in their shared bidirectional promoter. J. Immunol. 159:3068-3080.

10. Hayashi, T., and Faustman, D. 1999. NOD mice are defective in proteasome production and activation of NF-kB. Mol. Cell. Biol. 19:8646-8659.

11. Faustman, D., et al. 1991. Linkage of faulty major histocompatibility complex class I to autoimmune diabetes. Science. 254:1756-1761.

12. Markiewicz, M.A., et al. 1998. Long-term T cell memory requires the surface expression of self-peptide/major histocompatibility complex molecules. Proc. Natl. Acad. Sci. USA. 95:3065-3070.

13. Fu, Y., Nathan, D.M., Li, F., Li, X., and Faustman, D.L. 1993. Defective major histocompatibility complex class I expression on lymphoid cells in autoimmunity. J. Clin. Invest. 91:2301-2307.

14. Li, F., Hauser, S.L., Linan, M.J., Stein, M.C., and Faustman, D.L. 1995. Reduced expression of peptide-loaded HLA class I molecules on multiple sclerosis lymphocytes. Ann. Neurol. 38:147-154.

15. Palombella, V., Rando, O.J., Goldberg, A.L., and Maniatis, T. 1994. The ubiquitin-proteasome pathway is required for processing the NF-kB1 precursor protein and the activation of NF-kB. Cell. 78:773-785.

16. Coux, O., and Goldberg, A.L. 1998. Enzymes catalyzing ubiquitination and proteolytic processing of the 105 precursor of nuclear factor $\mathrm{kB} 1$.J. Biol. Chem. 273:8820-8828.

17. Sears, C., Olesen, J., Rubin, D., Finley, D., and Maniatis, T. 1998. NF-kB p105 processing via the ubiquitin-proteasome pathway. J. Biol. Chem. 273:1409-1419.

18. Beg, A.A., and Baltimore, D. 1996. An essential role for NF-kappaB in preventing TNF-alpha-induced cell death. Science. 274:782-784.

19. Van Antwerp, D.J., Martin, S.J., Kafri, T., Green, D.R., and Verma, I.M. 1996. Suppression of TNF-alpha-induced apoptosis by NF-kappaB. Science. 274:787-789.

20. Baeuerle, P.A., and Baltimore, D. 1996. NF-kappa B: ten years after. Cell. 87:13-20.

21. Faustman, D., Hauptfeld, V., Davie, J., Lacy, P., and Shreffler, D. 1980 Murine pancreatic $\beta$ cells express $\mathrm{H}-2 \mathrm{~K}$ and $\mathrm{H}-2 \mathrm{D}$ but not Ia antigens. J. Exp. Med. 151:1563-1569.

22. Vidal-Puig, A., and Faustman, D.L. 1994. Tolerance to peripheral tissue is transient and maintained by tissue specific class I expression. Transplant. Proc. 26:3314-3316.

23. Pestano, G.A., et al. 1999. Inactivation of misselected CD8 T cells by CD8 gene methylation and cell death. Science. 284:1187-1191.

24. Hyafil, F., and Strominger, J.L. 1979. Dissociation and exchange of the $\beta_{2-}$ microglobulin subunit of HLA-A and HLA-B antigens. Proc. Natl. Acad. Sci. USA. 76:5834-5838

25. Bernabeu, C., van de Rijn, M., Lerch, P.G., and Terhorst, C.P. 1984. $\beta_{2-}$ microglobulin from serum associates with MHC class I antigens on the surface of cultured cells. Nature. 308:642-645.

26. Hayashi, T., and Faustman, D. 2000. Essential role of human leukocyte antigen-encoded proteasome subunits in NF-kappaB activation and prevention of tumor necrosis factor-alpha- induced apoptosis. J. Biol. Chem. 275:5238-5247.

27. Hayashi, T., Kodama, S., and Faustman, D.L. 2000. Reply to 'LMP2 expression and proteasome activity in NOD mice'. Nat. Med. 6:1065-1066.

28. Jacob, C.O., Aiso, S., Michie, S. A., McDevitt, H.O, and Acha-Orbea, $\mathrm{H}_{\text {. }}$ 1990. Prevention of diabetes in nonobese diabetic mice by tumor necrosis factor (TNF): similarities between TNF-alpha and interleukin I. Proc Natl. Acad. Sci. USA. 87:968-972.

29. Ulaeto, D., Lacy, P.E., Kipnis, D.M., Kanagawa, O., and Unanue, E.R. 1992. A T-cell dormant state in the autoimmune process of nonobese diabetic mice treated with complete Freund's adjuvant. Proc. Natl. Acad. Sci. USA 89:3927-3931.

30. Qin, H.Y., Sadelain, M.W., Hitchon, C., Lauzon, J., and Singh, B. 1993. Complete Freund's adjuvant-induced $\mathrm{T}$ cells prevent the development and adoptive transfer of diabetes in nonobese diabetic mice. J. Immunol. 150:2072-2080.

31. Rabinovitch, A., Suarez-Pinzon, W.L., Lapchak, P.H., Meager, A., and Power, R.F. 1995. Tumor necrosis factor mediates the protective effect of Freund's adjuvant against autoimmune diabetes in BB rats. J. Autoimmun. 8:357-366

32. Rabinovitch, A., Suarez-Pinzon, W.L., Sorensen, O., Rajotte, R.V., and Power, R.F. 1997. TNF-alpha down-regulates type 1 cytokines and prolongs survival of syngeneic islet grafts in nonobese diabetic mice. $J$ Immunol. 159:6298-6303.

33. Gazda, L.S., Charlton, B., and Lafferty, K.J. 1997. Diabetes results from a late change in the autoimmune response of NOD mice. J. Autoimmun. 10:261-270.

34. Trowsdale, J., et al. 1990. Sequences encoded in the class II region of the $\mathrm{MHC}$ related to the 'ABC' superfamily of transporters. Nature. 348:741-744

35. Robertson, R.P., Zhang, H.J., Pyzdrowski, K.L., and Walseth, T.F. 1992 Preservation of insulin mRNA levels and insulin secretion in HIT cells by avoidance of chronic exposure to high glucose concentrations. J. Clin. Invest. 90:320-325.

36. Juang, J.H., Bonner-Weir, S., Wu, Y.J., and Weir, G.C. 1994. Beneficial influence of glycemic control upon the growth and function of transplanted islets. Diabetes. 43:1334-1339.

37. Lanza, R.P., et al. 1992. Transplantation of encapsulated canine islets into spontaneously diabetic $\mathrm{BB} /$ Wor rats without immunosuppression. Endocrinology. 131:637-642.

38. Faustman, D., Schoenfeld, D., and Ziegler, R. 1991. T-lymphocyte changes linked to autoantibodies: association of insulin autoantibodies with CD4+CD45R+ lymphocyte subpopulation in prediabetic subjects. Dia betes. 40:590-597.

39. Schatz, D.A., Riley, W.J., Maclaren, N.K., and Barrett, D.J. 1991. Defective inducer $\mathrm{T}$ cell function before the onset of insulin-dependent diabetes mellitus. J. Autoimmun. 4:125-136.

40. Peakman, M., Mahaligam, M., Leslie, R.D., and Vergani, D. 1993. Coexpression of CD45 RA (naive) and CD45RO (memory) T-cell markers. Lancet. 342:1296.

41. Sreenan, S., et al. 1999. Increased beta-cell proliferation and reduced mass before diabetes onset in the nonobese diabetic mouse. Diabetes. 48:989-996. 